\title{
GENERAL REPRESENTATION THEORY OF JORDAN ALGEBRAS
}

\author{
BY \\ N. JACOBSON
}

The theory of Jordan algebras has originated in the study of subspaces of an associative algebra that are closed relative to the composition $a b=a \times b$ $+b \times a$ where the $\times$ denotes the associative product. Such systems are called special Jordan algebras. It is well known that the composition $a b$ satisfies the conditions

$$
a b=b a, \quad\left(a^{2} b\right) a=a^{2}(b a) .
$$

This has led to the definition of an (abstract) Jordan algebra as a (nonassociative) algebra whose multiplication satisfies the above conditions. It is an open question as to how extensive is the subclass of special Jordan algebras in the class of Jordan algebras. However, it is known that there exist Jordan algebras which are not special.

If $\mathfrak{A}$ is a special Jordan algebra, then it is natural to consider the linear mappings $a \rightarrow U_{a}$ of $\mathfrak{A}$ into linear transformations $U_{a}$ such that

$$
U_{a b}=U_{a} U_{b}+U_{b} U_{a}
$$

for these mappings are just the homomorphisms of $\mathfrak{A}$ into special Jordan algebras of linear transformations. We shall now call such mappings special representations of $\mathfrak{A}$. Special representations have been considered previously by $\mathrm{F}$. D. Jacobson and the present author and complete results have been obtained for finite-dimensional semi-simple algebras of characteristic $0\left({ }^{1}\right)$.

There is another mapping of $\mathfrak{A}$ into linear transformations which is fundamental in the structure theory of Jordan algebras, namely, the regular mapping $a \rightarrow R_{a}$ where $R_{a}$ is the multiplication $x \rightarrow x a=a x$. It is known that the $R_{a}$ satisfy the following functional equations:

$$
\left[R_{a} R_{b c}\right]+\left[R_{b} R_{a c}\right]+\left[R_{c} R_{a b}\right]=0
$$

where, as usual, $[A B]$ denotes $A B-B A$ and

$$
R_{a} R_{b} R_{c}+R_{c} R_{b} R_{a}+R_{(a c) b}=R_{a} R_{b c}+R_{b} R_{a c}+R_{c} R_{a b}\left({ }^{2}\right)
$$

Presented as an invited address in the Algebra Conference of the International Congress of Mathematicians, September 2, 1950; received by the editors August 17, 1950.

(1) [7] A (special) representation in the sense of [7] as a linear mapping $a \rightarrow V_{a}, V_{a}$ a linear transformation, such that $V_{a b}=\left(V_{a} V_{b}+V_{b} V_{a}\right) / 2$. If $V_{a}$ satisfies this condition, then $U_{a}=V_{a} / 2$ satisfies $(0.1)$. The change to $(0 . \hat{1})$ is made so that special representations will be representations in the sense defined below. Numbers in brackets refer to the bibliography at the end of the paper.

(2) Cf. [1, p. 549]. 
As a generalization of this situation we define a (general) representation of $\mathfrak{A}$ to be a linear mapping $a \rightarrow S_{a}$ of $\mathfrak{A}$ into linear transformations $S_{a}$ which satisfy the functional equation for the $R$ 's. It is noteworthy that any special representation is a representation. Thus, the theory of representations encompasses both aspects of the theory of Jordan algebras that we have mentioned above. If $\mathfrak{A}$ is a subalgebra of a larger algebra $\mathfrak{B}$, then it is clear that the correspondence $a \rightarrow R_{a}$, where $R_{a}$ now denotes the multiplication in $\mathfrak{B}$ by $a \in \mathfrak{A}$, is a representation of $\mathfrak{A}$. It is clear from this remark that the theory of representations will be a basic tool in the study of subalgebras of Jordan algebras.

The main objective of the present paper is the development of the general representation theory of Jordan algebras. The only application which we shall note explicitly here is to the study of semi-simple subalgebras of an arbitrary finite-dimensional Jordan algebra of characteristic 0 . In this connection we obtain the perfect analogues of the results of Malcev and of Harish-Chandra on the theory of the Levi decomposition of a Lie algebra $[12 ; 5]$.

An important incidental tool in our discussion is another type of abstract system called a Lie triple system. A special Lie triple system is defined to be a subspace of an associative algebra which is closed under the ternary composition $[[a b] c]$. It is easy to see that any special Jordan algebra is a special Lie triple system. Contrary to the situation which obtains for special Jordan algebras, it is possible (as will be shown below) to give a perfect axiomatic description of special Lie triple systems. The corresponding abstract systems-(abstract) Lie triple systems-play an important role also in the theory of abstract Jordan algebras. Thus, we can show that any Jordan algebra $\mathfrak{A}$ is a Lie triple system relative to the composition $[a b c] \equiv(b c) a$ $-b(c a)$. Any representation of $\mathfrak{A}$ is a representation of the associator Lie triple system $\mathfrak{A}$. On the other hand, any Lie triple system can be imbedded in a Lie algebra in such a way that a representation of the Lie triple system can be extended to one of the Lie algebra. This observation will enable us to apply the well developed theory of Lie algebras to the present problem. The incidental results which we obtain on Lie triple systems may also be of some intrinsic interest, since these systems constitute a generalization of Lie algebras as well as of Jordan algebras.

\section{General theORY}

1. Jordan algebras and Lie triple systems. A (nonassociative) algebra $\mathfrak{A}$ over a field $\Phi$ is called an (abstract) Jordan algebra if the multiplication composition satisfies the following identities:

$$
\begin{aligned}
a b & =b a, \\
\left(a^{2} b\right) a & =a^{2}(b a) .
\end{aligned}
$$

We write $F(a)=\left(a^{2} b\right) a-a^{2}(b a)$ and form the second difference 


$$
\begin{aligned}
\Delta^{2} F= & F(x+y+z)-F(x+y)-F(y+z)-F(x+z) \\
& +F(x)+F(y)+F(z) .
\end{aligned}
$$

If the characteristic is not equal to $2, \Delta^{2} F=0$ gives

$$
x y b z+y z b x+z x b y=(x y)(b z)+(y z)(b x)+(z x)(b y),
$$

where we have abbreviated $\left.\left(\left(a_{1} a_{2}\right) a_{3}\right) a_{n}\right)$ to $a_{1} a_{2} \cdots a_{n}$. Conversely, if the characteristic of the base field is not equal to 3 , then (1.3) implies (1.2). From now on we shall assume that the characteristic is not equal to 2 or 3 . If we denote the mapping $x \rightarrow x a(=a x)$ by $R_{a}$ and change the notation slightly, then from (1.3) we obtain the relations

$$
\begin{aligned}
{\left[R_{a} R_{b c}\right]+\left[R_{b} R_{c a}\right]+\left[R_{c} R_{a b}\right] } & =0, \\
R_{a} R_{b} R_{c}+R_{c} R_{b} R_{a}+R_{(a c) b} & =R_{a} R_{b c}+R_{b} R_{c a}+R_{c} R_{a b} .
\end{aligned}
$$

We remark that either (1.4) or (1.5) and the commutative law imply (1.3). If we interchange $a$ and $b$ in (1.5) and subtract, we obtain the important relation

$$
\left[\left[R_{\alpha} R_{b}\right] R_{c}\right]=R_{A(b, c, a)},
$$

where $A(b, c, a)$ is the associator $(b c) a-b(c a)$.

We define next an (abstract) Lie triple system. This is a vector space over a field $\Phi$ in which a ternary composition $[a b c]$ is defined which is trilinear and satisfies:

$$
\begin{aligned}
{[a a b] } & =0, \\
{[a b c]+[b c a]+[c a b] } & =0, \\
{[[a b c] d e]+[[b a d] c e]+[b a[c d e]]+[c d[a b e]] } & =0, \\
{[[a b c] d e]+[[b a d] c e]+[[d c b] a e]+[[c d a] b e] } & =0, \\
{[[[a b c] d e] f g]+[[[b a e] d f] e g] } & \\
+[[[b a d] c e] f g]+[[[a b d] c f] e g]+Q+R & =0,
\end{aligned}
$$

where $Q$ and $R$ are obtained from the four previous terms in (1.11) by cyclic permutation of the pairs $(a, b),(c, d),(e, f)$.

If $\mathfrak{Z}$ is a Lie algebra and we defined $[a b c]=[[a b] c]$ in terms of the Lie composition $[a b]$, then $\mathbb{R}$ is a Lie triple system relative to $[a b c][10$, p. 152]. In particular any associative algebra is a Lie triple system relative to $[a b c]$ $=[[a b] c]$ where $[a b]=a b-b a$.

We shall now show that any Jordan algebra is a Lie triple system relative to the composition $[a b c] \equiv A(b, c, a)$. We assume first that $\mathfrak{A}$ has an identity. In this case the correspondence $a \rightarrow R_{a}$ is $1-1$. By (1.6),

$$
R_{[a b c]}=\left[R_{a} R_{b} R_{c}\right] \equiv\left[\left[R_{a} R_{b}\right] R_{c}\right] .
$$


Since $\left[R_{a} R_{b} R_{c}\right]$ satisfies (1.7)-(1.11) the same holds for $[a b c]$. Hence our assertion is proved. If $\mathfrak{A}$ does not have an identity, then we can adjoin one in the usual fashion. The result is a Jordan algebra. We can now use the $R_{a}$ in the extended algebra to prove that $\mathfrak{A}$ is a Lie triple system relative to $[a b c]$. We shall call this Lie triple system the associator system of $\mathfrak{A}$.

\section{Definition and elementary properties of representations.}

Definition 2.1. A linear mapping $a \rightarrow S_{a}$ of a Jordan algebra $\mathfrak{A}$ into the algebra of linear transformations of a vector space $\mathfrak{M}$ over $\Phi$ is called a representation if

$$
\begin{aligned}
{\left[S_{a} S_{b c}\right]+\left[S_{b} S_{c a}\right]+\left[S_{c} S_{a b}\right] } & =0 \\
S_{a} S_{b} S_{c}+S_{c} S_{b} S_{a}+S_{(a c) b} & =S_{a} S_{b c}+S_{b} S_{c a}+S_{c} S_{a b}
\end{aligned}
$$

This concept is equivalent to that of a module which has been introduced by Eilenberg [4, p. 133]. We define the latter in the following definition.

Definition 2.2. A Jordan module is a system consisting of a vector space $\mathfrak{M}$, a Jordan algebra $\mathfrak{A}$, and two compositions $x a, a x$ for $x$ in $\mathfrak{M}, a$ in $\mathfrak{A}$ which are bilinear and satisfy

$$
\begin{aligned}
a x & =x a, \\
(x a)(b c)+(x b)(c a)+(x c)(a b) & =(x(b c)) a+(x(c a)) b+(x(a b)) c, \\
x a b c+x c b a+a c b x & =(x a)(b c)+(x b)(c a)+(x c)(a b) .
\end{aligned}
$$

(As for algebras, $a_{1} a_{2} \cdots a_{n}$ stands for $\left(\left(a_{1} a_{2}\right) \cdots a_{n}\right)$.)

If $S: a \rightarrow S_{a}$ is a representation acting in the vector space $\mathfrak{M}$, then we obtain a module by setting $x a=a x=x S_{a}$. Conversely, if a module is given and $S_{a}$ is defined to be the mapping $x \rightarrow x a$, then $a \rightarrow S_{a}$ is a representation.

The relations (1.4) and (1.5) show that the correspondence $a \rightarrow R_{a}$ is a representation. We call this representation the regular representation. The corresponding module consists of $\mathfrak{A}, \mathfrak{A}$, and the multiplication composition defined in $\mathfrak{A}$. More generally if $\mathfrak{A}$ is a subalgebra of an algebra $\mathfrak{B}$, then $a \rightarrow R_{a}$ (acting in $\mathfrak{B}$ ) is a representation. Also the contraction of a representation to a submodule and the induced mapping in a difference module are representations. In particular, if $\mathfrak{A}$ is a subalgebra of $\mathfrak{B}$ and $\mathfrak{Y}$ is an ideal in $\mathfrak{B}$, then the contractions of the $R_{a}$ to $\mathfrak{Y}$ define a representation. Conversely any representation can be obtained in this way.

Thus, let $\mathfrak{M}$ be a Jordan module for $\mathfrak{A}$. Let $\mathfrak{B}=\mathfrak{A} \oplus \mathfrak{M}$ and define a product in $\mathfrak{B}$ by the rule

$$
\left(a_{1}+m_{1}\right)\left(a_{2}+m_{2}\right)=a_{1} a_{2}+a_{1} m_{2}+m_{1} a_{2}
$$

where $a_{i} \in \mathfrak{A}, m_{i} \in \mathfrak{M}$. Then $\mathfrak{B}$ is a commutative algebra. Also the module conditions insure that (1.3) holds if any one of the arguments is in $\mathfrak{M}$ and the remaining ones are in $\mathfrak{A}$. Finally since $m_{1} m_{2}=0,(1.3)$ holds trivially if two or 
more arguments are in $\mathfrak{M}$. Hence (1.3) holds in $\mathfrak{B}$ and $\mathfrak{B}$ is a Jordan algebra. Now it is clear that $\mathfrak{M}$ is an ideal in $\mathfrak{A}$ and that the contraction of $R_{a}, a$ in $\mathfrak{A}$, to $\mathfrak{M}$ is the $S_{a}$ determined by the module $\mathfrak{M}$. We shall call the Jordan algebra $\mathfrak{B}$ the semi-direct sum of $\mathfrak{A}$ and the module $\mathfrak{M}$.

As we have noted in the introduction, any associative algebra is a Jordan algebra relative to the composition $\{a b\}=a b+b a$. It follows that a mapping $a \rightarrow U_{a}$ of a Jordan algebra $\mathfrak{A}$ into the algebra of linear transformations of a vector space is a homomorphism into the special Jordan algebra of these transformations provided that $a \rightarrow U_{a}$ is linear and

$$
U_{a b}=U_{a} U_{b}+U_{b} U_{a} .
$$

A direct verification, which we omit, shows that $a \rightarrow U_{a}$ is a representation in the present sense. We shall call representations of this type special.

Next let $U$ and $V$ be two special representations acting in the same vector space. Assume that these commute in the sense that $\left[U_{a} V_{b}\right]=0$ for all $a$ and $b$. Set $S_{a}=U_{a}+V_{a}$. Another simple verification, which we also omit, shows that $a \rightarrow S_{a}$ is a representation.

In particular if $a \rightarrow U_{a}$ and $a \rightarrow V_{a}$ are arbitrary special representations, then $a \rightarrow U_{a} \times 1+1 \times V_{a}$ is a representation. We call this representation the Kronecker sum of the given special representations.

Next let $a \rightarrow \theta(a)$ be a homomorphism of $\mathfrak{A}$ into a second Jordan algebra $\mathfrak{B}$ and let $S$ be a representation of $\mathfrak{B}$. Define $T_{a}=S_{\theta(a)}$. Then it is clear that $a \rightarrow T_{a}$ is a representation.

As in the special case of the regular representation, we can derive from (2.2) the relation

$$
S_{[a b c]}=\left[\left[S_{a} S_{b}\right] S_{c}\right]
$$

This equation shows that any representation of a Jordan algebra is also a representation of the associator Lie triple system, that is, it is a homomorphism of the associator system into the special Lie triple system of linear transformations.

If $S$ is a homomorphism of a Lie triple system $\mathfrak{T}$, then the kernel $\Omega$ of $S$ is a subspace of $\mathfrak{T}$ which has the property that $[a b c] \in \Omega$ if any one of the factors $a, b$, or $c$ is in $\Re$. A subspace of a Lie triple system which has this closure property is called an ideal. If $\mathfrak{A}$ is a Jordan algebra and $\Re$ is an ideal in the associator system of $\mathfrak{A}$, then $\Re+\Re \mathfrak{A}$ is a Jordan ideal. For, if $z \in \Omega$ and $a_{1}$ and $a_{2}$ are arbitrary, then $\left(z a_{1}\right) a_{2}=A\left(z, a_{1}, a_{2}\right)+z\left(a_{1} a_{2}\right) \in \Omega+\Omega \Re$. In particular if $S$ is an arbitrary representation of $\mathfrak{A}$ and $\Omega$ is the kernel, then $\Re+\Omega \Re$ is an ideal. Of course, if $S$ is special then $\Omega$ itself is a Jordan ideal.

If $\mathfrak{A}$ is an algebra with an identity, then a special role is played by the representations $S$ such that $S_{1}=1$. For the corresponding module we have the condition $x 1=x=1 x$. We note that if $S$ is special then $S_{1}=2 S_{1}^{2}$ so that in this case $S_{1} \neq 1$. 
3. Universal associative algebras. The subalgebra generated by a subset of an associative (Lie) algebra will be called the enveloping associative (Lie) algebra of the subset. The enveloping associative (Lie) algebra of the set of representing transformations $S_{a}$ of a representation $S$ is called the enveloping associative (Lie) algebra of $S$. In this section we consider associative algebras and we define certain universal algebras which are the most general enveloping algebras for the complete set of representations and for certain subsets of the set of representations.

Let $\mathfrak{F}$ be the free associative algebra based on the vector space $\mathfrak{A}$. Thus, $\mathfrak{F}$ is the direct sum of $\mathfrak{A}$ and the Kronecker product spaces $\mathfrak{A} \times \mathfrak{A}$, $\mathfrak{U} \times \mathfrak{A} \times \mathfrak{A}, \cdots$ and multiplication in $\mathfrak{F}$ is the bilinear composition $(X)$ such that

$$
\left(a_{1} \times \cdots \times a_{r}\right) \times\left(a_{r+1} \times \cdots \times a_{s}\right)=a_{1} \times \cdots \times a_{r} \times a_{r+1} \times \cdots \times a_{s} .
$$

Let $\Re$ be the ideal in $\mathfrak{F}$ generated by the elements

$$
\begin{gathered}
a \times b c-b c \times a+b \times a c-a c \times b+c \times a b-a b \times c, \\
a \times b \times c+c \times b \times a+(a c) b-a \times b c-b \times c a-c \times a b,
\end{gathered}
$$

and let $\mathfrak{U}$ be the difference algebra $\mathfrak{F} / \Re$. We denote the coset $a+\Re$ of $a \in \mathfrak{A}$ by $\bar{a}$ and we denote the set of $\bar{a}$ 's by $\overline{\mathfrak{A}}$. Then $\mathfrak{U}$ is generated by $\overline{\mathfrak{A}}$. We simplify our notation by writing products in $\mathfrak{U}$ by $\bar{a} \bar{b}(a, b$ in $\mathfrak{U})$, and so forth, in place of the more accurate notation $\bar{a} \times \bar{b}$, and so forth. Then by (3.1) and (3.2) we have the relations

$$
\begin{aligned}
{[\bar{a}, \overline{b c}]+[\bar{b}, \overline{a c}]+[\bar{c}, \overline{a b}] } & =0, \\
\bar{a} \bar{b} \bar{c}+\bar{c} \bar{b} \bar{a}+\overline{(a c) b} & =\bar{a} \overline{b c}+\bar{b} \bar{c} \bar{a}+\bar{c} \overline{a b}
\end{aligned}
$$

for $\bar{a}, \bar{b}, \bar{c}$ in $\overline{\mathfrak{A}}$.

Now suppose that $S$ is a representation of $\mathfrak{A}$. Then the mapping $a \rightarrow S_{a}$ defines a unique homomorphism of the algebra $\mathfrak{F}$ onto the enveloping algebra $\mathfrak{E}_{S}$ of $S$. The defining conditions show that the kernel $\mathfrak{\Omega}_{S}$ of this homomorphism contains the ideal $\Omega$. It follows that the mapping $a \rightarrow S_{a}$ defines a unique representation of the associative algebra $\mathfrak{U}$. Conversely if we have a representation of $\mathfrak{u}$, then we can define $S_{a}$ to be the image of $\bar{a}$ under this representation. Then it is evident that $a \rightarrow S_{a}$ is a representation of the Jordan algebra. Since every associative algebra has a 1-1 representation by linear transformations, it is clear that there exist representations $S$ of $\mathfrak{A}$ for which the kernel $\Omega_{S}=\Re$. Thus, the algebra $\mathfrak{U}$ is the maximal enveloping algebra for the representations. For this reason we shall call $\mathfrak{U}$ the universal associative algebra (of the representations) of $\mathfrak{A}$.

It is easy to see that every Jordan algebra has a 1-1 representation. Thus, if $\mathfrak{A}$ has an identity, then the regular representation is $1-1$. If $\mathfrak{A}$ does not have an identity, then we can take the regular representation in the 
algebra obtained by adjoining an identity. The existence of a 1-1 representation implies that the mapping $a \rightarrow \bar{a}$ of $\mathfrak{A}$ onto $\overline{\mathfrak{A}}$ is $1-1$.

If $\mathfrak{A}$ is a special Jordan algebra, then the concept of the universal algebra for the special representations has been defined before $[7$, p. $144 ; 3$, p. 117]. We can also define this algebra for any Jordan algebra. As before, we form the free algebra $\mathfrak{F}$ and we consider the difference algebra $\mathfrak{H}_{s}=\mathfrak{F} / \mathfrak{\Omega}^{(s)}$ where $\Omega^{(s)}$ is the ideal generated by the elements $a \times b+b \times a-a b$. This time we denote the coset of $a \in \mathfrak{A}$ by $a_{s}$. Then the set of $a_{s}$ generates $\mathfrak{U}_{s}$ and we have the relations

$$
(a b)_{s}=a_{s} b_{s}+b_{s} a_{s}
$$

for $a, b$ in $\mathfrak{A}$. Results similar to those which we have stated for $\mathfrak{U}$ and arbitrary representations hold for $\mathfrak{U}_{s}$ and special representations. In particular, any special representation can be extended to a representation of $\mathfrak{U}_{s}$. The mapping $a \rightarrow a_{s}$ is $1-1$ if and only if $\mathfrak{A}$ is special. We shall call $\mathfrak{U}_{s}$ the special universal associative algebra of $\mathfrak{A}$.

Next let $\mathfrak{U}_{s}^{*}$ be the algebra obtained by adjoining a new identity 1 to $\mathfrak{U}_{s}$. Consider the Kronecker product $\mathfrak{U}_{s}^{*} \times \mathfrak{u}_{s}^{*}$ and let $\mathfrak{U}_{s}^{(2)}$ be the subalgebra generated by the elements $a_{s}^{(2)}=a_{s} \times 1+1 \times a_{s}, a$ in $\mathfrak{A}$. We shall call $\mathfrak{U}_{s}^{(2)}$ the Kroncker sum of $\mathfrak{U}_{s}$ with itself. If $S$ is a representation that is a Kronecker sum $U+V$ of special representations $U$ and $V$, then it is easy to see that the mapping $a_{s}^{(2)} \rightarrow S_{a}$ can be extended to a representation of $\mathfrak{U}_{s}^{(2)}$. Also there exist $S=U+V$ for which the indicated homomorphism is an isomorphism. In this sense $\mathfrak{U}_{s}^{(2)}$ is universal for the Kronecker sums of special representations.

If $\mathfrak{A}$ is a Jordan algebra with an identity, we can define still another universal associative algebra, namely, the universal algebra for the representations $S$ such that $S_{1}=1$. This algebra can be defined as the algebra $\mathfrak{U}_{1}=\mathfrak{F} / \Re_{1}$ where $\Omega_{1}$ is the ideal generated by the elements (3.1) and (3.2) and the element $a \times 1-a, 1 \times a-a$. In this case we denote the coset of $a \in \mathfrak{A}$ by $\tilde{a}$. If $S$ is a representation such that $S_{1}=1$, then there exists a homomorphism of $\mathfrak{U}_{1}$ onto the enveloping associative algebra of $S$. Also there exists an $S$ such that $S_{1}=1$ and such that this homomorphism is an isomorphism.

It is clear from the above discussion that the correspondences $\bar{a} \rightarrow a_{s}$, $\bar{a} \rightarrow a_{s}^{(2)}, \bar{a} \rightarrow \tilde{a}$ define homomorphisms of $\mathfrak{U}$ onto $\mathfrak{U}_{s}, \mathfrak{U}_{s}^{(2)}$, and $\mathfrak{U}_{1}$ respectively. Also since any special representation can be regarded as the Kronecker sum of itself and the 0 representation, $a_{s}^{(2)} \rightarrow a_{s}$ defines a homomorphism.

4. Universal associative algebra of a Jordan algebra with an identity. Let $a$ be an element of an arbitrary Jordan algebra and let $\bar{a}$ be the corresponding element of the universal associative algebra $\mathfrak{U}$. Then by (3.3) and (3.4) we have

$$
\begin{gathered}
\bar{a} \overline{a^{2}}=\overline{a^{2}} \bar{a}, \\
\overline{a^{r}}=\overline{a^{r-2}} \overline{a^{2}}+2 \bar{a} \overline{a^{r-1}}-\overline{a^{r-2}} \bar{a}^{2}-\bar{a}^{2} \overline{a^{r-2}}, \quad r \geqq 3 .
\end{gathered}
$$


Thus, $\bar{a}$ and $\overline{a^{2}}$ generate a commutative algebra and $\overline{a^{r}}$ belongs to this algebra. Now (4.2) can be simplified to

$$
\overline{a^{r}}=2 \bar{a} \overline{a^{r-1}}-\left(2 \bar{a}^{2}-\overline{a^{2}}\right) \overline{a^{r-2},} \quad r \geqq 3 .
$$

If we set $\overline{a^{r}}=f(r), A=f(1), B=f(2)$ then (4.3) becomes

$$
f(r)=2 A f(r-1)-\left(2 A^{2}-B\right) f(r-2), \quad r \geqq 3 .
$$

This recursion formula can be solved and one obtains $\left({ }^{3}\right)$

$$
f(r)=\frac{1}{2}\left\{\left(A+\left(B-A^{2}\right)^{1 / 2}\right)^{r}+\left(A-\left(B-A^{2}\right)^{1 / 2}\right)^{r}\right\}, r=1,2, \cdots .
$$

It is well known that any Jordan algebra is power associative in the sense that the subalgebra generated by a single element is associative (see, for example, $[1$, p. 550]). Thus, powers are uniquely defined and if $\phi(\lambda)$ is a polynomial in an indeterminate $\lambda$, then $\phi(a)$ is uniquely defined. Now suppose that $a$ is algebraic, that is, there exists a polynomial $\phi(\lambda) \neq 0$ such that $\phi(a)=0$. Then if $\phi(\lambda)=\prod\left(\lambda-\rho_{i}\right)$ where $\rho_{i}$ are the roots of $\phi$ in a splitting field, and $\psi(\lambda)=\prod_{i \leqq 1}\left(\lambda-\left(\rho_{i}+\rho_{j}\right) / 2\right)$, the element $\bar{a}$ is algebraic and $\psi(\bar{a})=0$. In particular we see that if $e$ is an idempotent element of $A$ then

$$
\bar{e}(\bar{e}-1)(\bar{e}-1)=0\left({ }^{4}\right) .
$$

Assume now that $\mathfrak{A}$ has an identity 1. Then (4.6) holds for $e=1$. Also if we set $a=b=1$ in (3.3) we obtain $[\bar{c}, \overline{1}]=0$ and if we set $a=b=1$ in (3.4) we obtain

$$
\bar{c}=3 \overline{1} \bar{c}-2 \overline{1}^{2} \bar{c} .
$$

This shows that $3 \overline{1}-2 \overline{1}^{2}$ is an identity element in $\mathfrak{u}$. Accordingly we write $3 \overline{1}-2 \overline{1}^{2}=1$. Now set $E_{1}=2 \overline{1}^{2}-\overline{1}, E_{2}=4\left(\overline{1}-\overline{1}^{2}\right)$. Then, since $2 \overline{1}^{3}-3 \overline{1}^{2}+\overline{1}=0$, we can verify that

$$
E_{1}^{2}=E_{1}, \quad E_{2}^{2}=E_{2}, \quad E_{1} E_{2}=0=E_{2} E_{1}, \quad E_{1}+E_{2}=1 .
$$

It follows that $\mathfrak{U}=\mathfrak{u} E_{1} \oplus \mathfrak{u} E_{2}$.

We shall now show that $\mathfrak{u} E_{2}$ is essentially the special universal associative algebra $\mathfrak{U}_{s}$ and that $\mathfrak{u} E_{1}$ is essentially the same as $\mathfrak{U}_{1}$. We note first that the equation for $\overline{1}$ gives $\overline{1} E_{1}=E_{1}, \overline{1} E_{2}=E_{2} / 2$. Now consider the homomorphism $\bar{a} \rightarrow a_{s}$ of $\mathfrak{U}$ onto $\mathfrak{U}_{s}$. Since $2\left(1_{s}\right)^{2}-1_{s}=0, E_{1}$ is mapped into 0 . Hence the kernel of the homomorphism contains $\mathfrak{U} E_{1}$. Consequently we have a homomorphism of $\mathfrak{u} E_{2}$ onto $\mathfrak{U}_{s}$ sending $a E_{2}$ into $a_{s}$. On the other hand, if we set $a=1$ in (3.4)

(3) This formula and the formula for $\psi(\lambda)$ given below will be proved in a forthcoming paper by W. H. Mills which is to appear in the Pacific Journal of Mathematics.

(4) This can also be proved directly. Cf. [1, p. 550]. 
we obtain

$$
\overline{b c}-\bar{b} \bar{c}-\bar{c} \bar{b}=\overline{1}(\overline{b c}-\bar{b} \bar{c}-\bar{c} \bar{b}) .
$$

Multiplication by $E_{2}$ gives

$$
\overline{b c} E_{2}=\left(\bar{b} E_{2}\right)\left(\bar{c} E_{2}\right)+\left(\bar{c} E_{2}\right)\left(\bar{b} E_{2}\right) .
$$

Since $\mathfrak{U}_{s}$ is a universal algebra for special representations, it follows from this equation that $a_{8} \rightarrow \bar{a} E_{2}$ defines a homomorphism of $\mathfrak{U}_{s}$ onto $\mathfrak{u} E_{2}$. Hence the extension of the mapping $\bar{a} E_{2} \rightarrow a_{s}$ is an isomorphism of $\mathfrak{U} E_{2}$ onto $\mathfrak{U}_{8}$. Similarly, we can prove that the mapping $\bar{a} E_{1} \rightarrow \tilde{a}$ can be extended to an isomorphism of $\mathfrak{u} E_{1}$ onto $\mathfrak{U}_{1}$. We therefore have the following theorem.

THEOREM 4.1. The universal associative algebra of a Jordan algebra with an identity is isomorphic to a direct sum of the special universal algebra and the universal algebra for the representations $S$ for which $S_{1}=1$.

5. Imbedding of Lie triple systems in Lie algebras. As we shall show later, the theory of Lie triple systems plays an important role in the study of the representations of Jordan algebras. We have defined a Lie triple system as a vector space $\mathfrak{T}$ in which a ternary trilinear composition $[a b c]$ is defined satisfying (1.7)-(1.11). If $\mathfrak{T}$ is a subspace of a Lie algebra closed relative to $[[a b], c]$, then $\mathfrak{T}$ is a Lie triple system relative to $[a b c] \equiv[[a b] c]$. We shall show in this section that every Lie triple system can be obtained in this way.

Thus let $\mathfrak{I}$ be an arbitrary Lie triple system over a field of characteristic not 2. We consider the Kronecker product $\mathfrak{T} \times \mathfrak{T}$ and let $\Omega$ be the subset of vectors $\sum a \times b$ that have the property that $\sum[a b x]=0$ for all $x$ in $\mathfrak{T}$. It is clear that $\Omega$ is a subspace. Hence we can form the factor space $\overline{\mathfrak{T} \times \mathfrak{T}}$ of $\mathfrak{T} \times \mathfrak{T}$ relative to $\Omega$. We shall now show that the vector space $\mathfrak{R}=\mathfrak{T} \oplus \overline{\mathfrak{T}} \times \mathfrak{T}$ can be made into a Lie algebra in such a way that the given composition $[a b c]$ in $\mathfrak{T}$ coincides with the composition $[[a b] c]$ defined in $\mathfrak{R}$.

If $a$ and $b \in \mathfrak{T}$ we define

$$
[a b]=\overline{a \times b} .
$$

Then any element of $\overline{\mathfrak{T} \times \mathfrak{T}}$ can be written as a sum $\sum[a b]$. We now define

$$
\begin{aligned}
{\left[\sum[a b], c\right] } & =\sum[a b c], \\
{\left[c, \sum[a b]\right] } & =-\sum[a b c], \\
{\left[\sum[a b], \sum[c d]\right] } & =\sum[[a b c], d]-\sum[[a b d], c] .
\end{aligned}
$$

We have to show first that (5.2)-(5.4) define single-valued compositions. It suffices to show that the right-hand side is 0 if either factor on the left is 0 . Thus suppose that $\sum[a b]=0$. Then $\sum a \times b \in \Omega$ and by definition $\sum[a b c]$ $=0$. In a similar fashion the other conditions can be established. 
We now define a composition $[u v]$ in $\mathbb{R}$ by specifying that if $u=a+\sum[b c]$, $v=d+\sum[e f]$, then

$$
[u v]=[a d]+\sum[a,[e f]]+\sum[[b c], d]+\sum[[b c],[e f]] .
$$

Then this multiplication is single-valued and bilinear. To prove the skew symmetry we have to show that

$$
[a b]=-[b a] \text { and }[[a b],[c d]]=-[[c d],[a b]] .
$$

The first of these is an immediate consequence of $[a b x]=-[b a x]$ which follows from (1.7). The second is that

$$
[[a b c], d]-[[a b d], c]+[[c d a], b]-[[c d b], a]=0 .
$$

This is equivalent to

$$
[[a b c] d x]-[[a b d] c x]+[[c d a] b x]-[[c d b] a x]=0
$$

for all $x$ in $\mathfrak{T}$. This follows from (1.10) and (1.7).

Next we have to verify Jacobi's identity. It suffices to prove this for elements that are either in $\mathfrak{T}$ or are of the form $[a b], a, b$ in $\mathfrak{T}$. Because of the skew symmetry we have to consider only four cases: all three elements in $\mathfrak{T}$, two in $\mathfrak{I}$ and one of the form $[a b]$, one in $\mathfrak{T}$ and two of the form $[a b]$, all three of the form $[a b]$. The first case is settled by referring to (1.8). To prove the second we note that

$$
\begin{aligned}
{[[[a b] c], d]+[[c d],[a b]] } & +[[d,[a b]], c] \\
& =[[a b c], d]+[[c d a], b]-[[c d b], a]-[[a b d], c]
\end{aligned}
$$

which is 0 by (1.10). Similarly (1.9) gives the Jacobi identity for $u=[a b]$, $v=[c d], w=c$ and (1.11) gives it for $u=[a b], v=[c d], w=[e f]$. Hence we have proved that $\mathfrak{R}$ is a Lie algebra. Moreover, it is clear from the definition (5.2) that the composition $[a b c]$ given in $\mathfrak{T}$ coincides with the Lie product $[[a b], c]$.

The Lie algebra which we have constructed out of the given Lie triple system need not give the most general imbedding of $\mathfrak{T}$. For example, let $\mathfrak{T}$ be the two-dimensional system with basis $x_{1}, x_{2}$ in which all the products $[a b c]$ are 0 . It is easy to see that the Lie algebra $\&$ is the two-dimensional $0 \mathrm{Lie}$ algebra. On the other hand we can obtain a more general imbedding of $\mathfrak{T}$ by constructing the Lie algebra $\mathfrak{M}$ with basis $x_{1}, x_{2}, x_{3}$ such that $\left[x_{1} x_{2}\right]=x_{3}$, $\left[x_{1} x_{3}\right]=0=\left[x_{2} x_{3}\right]$.

We now introduce the following definitions. If $\mathfrak{T}$ is a Lie triple system, then a mapping $a \rightarrow a^{T}$ of $\mathfrak{T}$ into a Lie algebra $\mathfrak{R}$ is called an imbedding of $\mathfrak{R}$ if (1) $\mathfrak{T}$ is linear and (2) $[a b c]^{T}=\left[\left[a^{T} b^{T}\right], c^{T}\right]$ holds for all $a, b, c$ in $\mathfrak{T}$. If $\mathfrak{T}$ is a Lie triple system contained in a Lie algebra in the sense that $\mathfrak{T}$ is a subspace of $\mathbb{R}$ closed relative to $[[a b], c]$, then it is easy to see that the enveloping Lie algebra of $\mathfrak{T}$ is $\mathfrak{T}+[\mathfrak{T} \mathfrak{T}]$, the set consisting of the elements of the form 
$a+\sum[b c]$ where $a, b, c \in \mathfrak{T}$. If $\mathfrak{T}$ is an imbedding of an abstract Lie triple system, then the enveloping Lie algebra of $\mathfrak{I}^{T}$ is called the enveloping Lie algebra of the imbedding. If $U$ and $T$ are imbeddings, then we say that $U$ is a cover of $T(U \geqq T)$ if the correspondence $a^{U} \rightarrow a^{T}$ is single-valued and can be extended to a homomorphism of the enveloping Lie algebra of $U$ onto that of $T$. The imbedding $U$ is universal if $U \geqq T$ for every imbedding $T$.

It is easy to prove the existence of a universal Lie algebra for a Lie triple system $\mathfrak{T}$ using the method which we employed for universal associative algebras. We form the free Lie algebra $\mathscr{S}_{L}$ over the vector space $T$. $\mathscr{S}_{L}$ is characterized by the following properties: (1) $\mathbb{S}_{L} \supseteq \mathfrak{T},(2)$ any linear transformation of $\mathfrak{T}$ into a Lie algebra can be extended to a homomorphism of $\$_{L}$. As has been shown by Witt [7, p. 155], $\mathfrak{S H}_{L}$ can be taken to be the Lie algebra which is obtained from the free associative algebra $\mathbb{B}$ over $\mathfrak{I}$ by defining $[x y] \equiv x \times y-y \times x$. Now let $\subseteq$ be the ideal in $\mathfrak{S}_{L}$ generated by the elements $[[a b] c]-[a b c], a, b, c$ in $\mathfrak{T}$. Then if $\bar{a}$ denotes the coset of $a \in \mathfrak{T}$, it is easily seen that $a \rightarrow \bar{a}$ is a universal imbedding of $\mathfrak{T}$.

We denote the universal imbedding of $\mathfrak{T}$ by $U$ and the imbedding which we constructed at the beginning of this section by $U^{\prime}$. Since $U^{\prime}$ is $1-1$ it follows that $U$ is $1-1$. Also we have the relation $\mathfrak{T}^{U^{\prime}} \cap\left[\mathfrak{I}^{U^{\prime}} \mathfrak{T}^{U^{\prime}}\right]=0$ in the enveloping Lie algebra of $U^{\prime}$ and this implies that $\mathfrak{I}^{U} \cap\left[\mathfrak{T}^{U} \mathfrak{T}^{U}\right]=0$.

II. REPRESENTATION THEORY FOR FINITE-DIMENSIONAL JORDAN ALGEBRAS

6. Finiteness of dimensionality of the universal algebras. In the remainder of this paper we restrict our attention to finite-dimensional algebras and to representations in finite-dimensional vector spaces. We prove first the following theorem.

THEOREM 6.1. The universal associative algebra of any Jordan algebra of finite dimension is finite-dimensional.

Proof. Equation (3.4) shows that if $\bar{a}, \bar{b}, \bar{c} \in \overline{\mathfrak{A}}$, then $\bar{a}^{3}$ and $\bar{a} \bar{b} \bar{a}$ are expressible in lower degree terms, that is, as sums of products of at most two $\bar{a} \in \overline{\mathfrak{A}}$. Also $\bar{a} \bar{b} \bar{c}$ and $-\bar{c} \bar{b} \bar{a}$ differ by terms of lower degree. In particular this holds for $\bar{a}^{2} \bar{b}$ and $-\bar{b} \bar{a}^{2}$. Now if $x_{1}, x_{2}, \cdots, x_{n}$ is a basis for $\mathfrak{A}$, then the cosets $\bar{x}_{1}, \bar{x}_{2}, \cdots, \bar{x}_{n}$ generate $\mathfrak{U}$. Let $\bar{y}$ be one of these and consider a monomial $\cdots \bar{y} \cdots \bar{y} \cdots$. We assert that if more than two $\bar{y}$ 's occur in the monomial, then we can express it as a linear combination of terms of lower degree. For, by the foregoing remarks, we can move any $\bar{y}$ two places to the left at the expense of lower degree terms. If the monomial has three $\bar{y}$ 's this leads either to a factor $\bar{y}^{3}$ or to a factor $\bar{y} \bar{x} \bar{y}$. In either case we obtain an expression in terms of monomials of lower degree. Hence we need consider only monomials in which each $\bar{x}_{i}$ occurs with multiplicity one or two. Since the number of such monomials is finite the theorem is proved.

We can obtain an upper bound to the dimensionality of $\mathfrak{U}$ as follows. We 
observe first that if there are two $\bar{y}$ 's in a monomial, then either this monomial can be expressed in lower terms or we can collect the $\bar{y}$ to obtain $\bar{y}^{2}$. Also we can place this term in any position at the expense of lower degree terms. Next we recall that a single $\bar{y}$ can be moved two places to the left or right. It follows that every element of $\mathfrak{U}$ is a linear combination of "standard" monomials

$$
\bar{x}_{k_{1}}^{2} \bar{x}_{k_{2}}^{2} \cdots \bar{x}_{k_{r}}^{2} \bar{x}_{k_{r+1}} \bar{x}_{k_{r+2}} \cdots \bar{x}_{k_{s}}
$$

where $k_{1}, k_{2}, \cdots, k_{s}$ are distinct elements of the range $1,2, \cdots, n$ and

$$
k_{1}<k_{2}<\cdots<k_{r} ; \quad k_{r+1}<k_{r+3}<\cdots ; k_{r+2}<k_{r+4}<\cdots .
$$

The number of ways of arranging $s-r$ numbers $k_{r+1}, \cdots, k_{s}$ so that the last two conditions hold is

$$
D_{s \rightarrow r}=C_{s-r,[(s-r) / 2], \quad D_{0}=1 .}
$$

It follows that the number of standard monomials does not exceed

$$
N=\sum_{s=1}^{n} \sum_{r=0}^{r} C_{n, s} C_{s, r} D(s-r) \text {. }
$$

Thus $\operatorname{dim} \mathfrak{U} \leqq N$. This bound is exact; for, it can be shown that if $\mathfrak{A}$ is the zero Jordan algebra with basis $x_{1}, x_{2}, \cdots, x_{n}$ and $x_{i} x_{j}=0$, then $\operatorname{dim} \mathfrak{U}=N$.

We have seen that the representations of the Jordan algebra $\mathfrak{A}$ are obtained from the representations of its universal algebra $\mathfrak{U}$. If we recall that a finite-dimensional associative algebra has only a finite number of inequivalent irreducible representations, we obtain the following corollary.

Corollary 6.1. Any finite-dimensional Jordan algebra has only a finite number of inequivalent irreducible representations.

The analogue of Theorem 6.1 holds also for Lie triple systems, that is, if $\mathfrak{T}$ is finite-dimensional, then the universal Lie algebra $\mathfrak{R}_{U}$ of $\mathfrak{T}$ is finite-dimensional. For $\mathfrak{R}_{U}=\mathfrak{T}^{U}+\left[\mathfrak{T}^{U} \mathfrak{T}^{U}\right]$. Hence since multiplication in $\mathfrak{R}_{U}$ is skew symmetric, $\operatorname{dim} \mathfrak{Q}_{U} \leqq n(n+1) / 2$ where $n=\operatorname{dim} \mathfrak{T}$.

7. Results on Lie triple systems. In this section we derive some results on Lie triple systems that will be required in the study of the structure of the enveloping association of Lie algebras of representations of Jordan algebras. The first result below is valid without restriction on the dimensionality or the characteristic; after this, however, we assume throughout that all vector spaces are finite-dimensional and that the base field has characteristic 0 .

THEOREM 7.1. If $\mathfrak{B}$ is an ideal in the Lie triple system $\mathfrak{T}$, then $\mathfrak{B}+[\mathfrak{B P}]$ is an ideal in the Lie algebra $\mathfrak{B}+[\mathfrak{B T}]$ and $\mathfrak{B}+[\mathfrak{B T}]$ is an ideal in $\mathfrak{T}+[\mathfrak{T} \mathfrak{T}]$.

Proof. We have the relation 
$[\mathfrak{B}+[\mathfrak{B P}], \mathfrak{B}+[\mathfrak{B T}]]=[\mathfrak{B P}]+[[\mathfrak{B P}], \mathfrak{B}]+[\mathfrak{B},[\mathfrak{B T}]]+[[\mathfrak{B P}],[\mathfrak{B T}]]$.

Also $[[\mathfrak{B R}],[\mathfrak{B T}]] \subseteq[\mathfrak{B},[\mathfrak{B}[\mathfrak{B T}]]+[\mathfrak{B},[\mathfrak{B}] \mathfrak{B T}]]$ by Jacobi's identity. Hence $[\mathfrak{B}+[\mathfrak{B R}], \mathfrak{B}+[\mathfrak{B T}]] \subseteq \mathfrak{B}+[\mathfrak{B R}]$. Similarly

$$
[\mathfrak{B}+[\mathfrak{B T}], \mathfrak{T}+[\mathfrak{T T}]]=[\mathfrak{B T}]+[\mathfrak{B}[\mathfrak{T T}]]+[[\mathfrak{B T}] \mathfrak{T}]+[[\mathfrak{B T}][\mathfrak{T T}]]
$$

and $[[\mathfrak{B T}],[\mathfrak{T T}]] \subseteq[[[\mathfrak{B T}] \mathfrak{T}] \mathfrak{T}] \subseteq[\mathfrak{B T}]$. Hence the right-hand side of the foregoing is contained in $\mathfrak{B}+[\mathfrak{B T}]$.

A subset $\mathfrak{B}$ of a Lie algebra $\mathfrak{R}$ is said to be subinvariant in $\mathbb{R}$ if there exists a chain $\mathbb{R}=\Omega_{1} \supseteq R_{2} \supseteq \cdots \supseteq \mathbb{R}_{m}=\mathfrak{B}$ such that each $\mathfrak{R}_{i}$ is an ideal in the preceding $\mathfrak{R}_{i-1}$ (cf. [15]). Thus Theorem 7.1 implies that the enveloping Lie algebra of $\mathfrak{B}$ is subinvariant in the enveloping Lie algebra of $\mathfrak{T}$. We prove next the following result which is a partial extension of a lemma of a former paper.

THEOREM 7.2. Let \& be an associative algebra of characteristic $0, \&$ a subalgebra of the Lie algebra $\mathfrak{E}_{L}, \mathfrak{B}$ a subinvariant subalgebra of $\mathbb{R}$. Then if the enveloping associative algebra $\mathfrak{B}^{*}$ of $\mathfrak{B}$ is nilpotent, $\mathfrak{B}$ is contained in the radical of the enveloping associative algebra $\mathfrak{R}^{*}$ of $\mathfrak{R}$.

Proof. We have the chain $\mathfrak{l}=\Omega_{1} \supseteq R_{2} \supseteq \cdots \supseteq R_{m}=\mathfrak{B}$ where $\Omega_{i}$ is an ideal in $\mathfrak{R}_{i-1}$. Assume that $\mathfrak{P}$ is in the radical of the enveloping associative algebra $\mathfrak{R}_{i}^{*}$ of $\mathfrak{R}_{i}$. Consider the mapping $x \rightarrow x a_{i-1}$ ] determined by any element $a_{i-1}$ of $\mathfrak{R}_{i-1}$. This mapping is a derivation which sends $\mathfrak{l}_{i}$ into itself. Since it is inner it is also a derivation in the enveloping associative algebra $\mathfrak{R}_{i}^{*}$ of $\mathfrak{R}_{i}$. Since the base field is of characteristic 0 , the radical $\Re\left(\Omega_{i}^{*}\right)$ of $\Omega_{i}^{*}$ is sent into itself by the derivation $\left[6\right.$, p. 692]. It follows that $\left[\Re\left(\mathfrak{R}_{i}^{*}\right), \mathfrak{R}_{i-1}\right] \subseteq \Re\left(\mathfrak{R}_{i}^{*}\right)$. Since $\mathfrak{P} \subseteq \Re\left(\mathfrak{R}_{i}^{*}\right)$ by assumption,

$$
\mathfrak{P}_{i-1} \equiv \mathfrak{P}+\left[\mathfrak{P R}_{i-1}\right]+\left[\left[\mathfrak{P R}_{i-1}\right] \mathfrak{R}_{i-1}\right]+\cdots \subseteq \Re\left(\mathfrak{R}_{i}^{*}\right) .
$$

It follows that the enveloping associative algebra $\mathfrak{P}_{i-1}^{*}$ is nilpotent. Since $\mathfrak{P}_{i-1}$ is an ideal in $\mathfrak{R}_{i-1}$ this implies that $\mathfrak{B}_{i-1}$ is in the radical of $\mathfrak{R}_{i-1}^{*}[8, \mathrm{p}$. $876]$. Hence $\mathfrak{B}$ is in the radical of $\mathfrak{R}_{i-1}^{*}$. The theorem now follows by induction.

We shall also require the following theorem.

THEOREM 7.3. Let $\mathfrak{T}$ be a Lie triple system of characteristic 0 and let $\mathfrak{I}$ be a 1-1 imbedding of $\mathfrak{T}$ such that (1) the enveloping Lie algebra $\mathfrak{R}_{T}$ is semi-simple and (2) $\mathfrak{I}^{T} \cap\left[\mathfrak{T}^{T} \mathfrak{I}^{T}\right]=0$. Then $\mathfrak{I}$ is a universal imbedding of $\mathfrak{T}$.

Proof. Let $U$ be a universal imbedding of $\mathfrak{T}$. Then the isomorphism $a^{U} \rightarrow a^{T}$ of $\mathfrak{I}^{T}$ onto $\mathfrak{T}^{U}$ can be extended to a homomorphism of $\mathfrak{Z}_{U}$ onto $\mathbb{R}_{T}$. We assert that the kernel of $\Omega$ of this homomorphism is the center $\mathbb{E}$ of $\Omega_{U}$. For, if $a^{U}+\sum\left[b^{U} c^{U}\right] \in \mathbb{C}$ then $a^{T}+\sum\left[b^{T} c^{T}\right]$ is in the center of $\mathfrak{R}_{T}$. Since $\mathfrak{R}_{T}$ is semi-simple, $a^{T}+\sum\left[b^{T} c^{T}\right]=0$; hence $a^{U}+\sum\left[b^{U} c^{U}\right]$ is in $\Omega$. Conversely let $a^{U}+\sum\left[b^{U} c^{U}\right] \in \Re$. Then $a^{T}+\sum\left[b^{T} c^{T}\right]=0$ and by (2), $a^{T}=0$ and $\sum\left[b^{T} c^{T}\right]$ $=0$. Hence $\sum\left[\left[b^{T} c^{T}\right] x^{T}\right]=0$ for all $x \in \mathfrak{T}$. This implies that $\sum\left[\left[b^{U} c^{U}\right] x^{U}\right]=0$. 
Since $a^{T}=0$ implies $a^{U}=0, a^{U}+\sum\left[b^{U} c^{U}\right]$ is in $\mathbb{6}$. Our assertion is therefore proved. We now have $\mathfrak{R}_{U} / \mathfrak{C} \cong \mathfrak{R}_{T}$. Since $\mathfrak{R}_{T}$ is semi-simple, it follows that $\mathfrak{C}$ is the radical of $\mathfrak{R}_{U}$. Hence by Levi's theorem $\mathfrak{R}_{U}=\mathfrak{\complement} \oplus \mathbb{S}$ where $\subseteq$ is a semisimple Lie algebra $\left.{ }^{5}\right)$. But $\mathfrak{R}_{T}=\mathfrak{T}^{T} \oplus\left[\mathfrak{T}^{T} \mathfrak{T}^{T}\right]$ is semi-simple; hence the derived algebra $\mathfrak{R}_{T}^{\prime}=\left[\left[\mathfrak{T}^{T \mathfrak{T}^{T}}\right] \mathfrak{T}^{T}\right]+\left[\mathfrak{T}^{T \mathfrak{T}^{T}}\right]=\mathfrak{R}_{T}\left({ }^{6}\right)$. It follows that $\mathfrak{T}^{T}$ $=\left[\left[\mathfrak{T}^{T \mathfrak{T}^{T}}\right] \mathfrak{T}^{T}\right]$ and this implies that $\mathfrak{T}^{U}=\left[\left[\mathfrak{T}^{U} \mathfrak{T}^{U}\right] \mathfrak{T}^{U}\right]$. Since $\mathfrak{R}_{U}=\mathfrak{T}_{U}$ $+\left[\mathfrak{T}_{U} \mathfrak{T}_{U}\right]$ the foregoing relation implies that $\mathfrak{R}_{U}^{\prime}=\mathfrak{R}_{U}$. Hence $\mathfrak{R}_{U}=\mathfrak{S}$, $\mathfrak{S}=0$, and the homomorphism of $\mathfrak{R}_{U}$ onto $\mathfrak{R}_{T}$ is an isomorphism.

8. Structure of the enveloping algebras of representations of Jordan algebras. We recall at this point the main concepts and results of the structure theory of Jordan algebras. We recall first that a Jordan algebra $\mathfrak{A}$ is solvable if $\mathfrak{A}^{2^{k}}=0$ for some integer $k$. Here $\mathfrak{A}^{2^{i}}=\mathfrak{A} 2^{2^{i-1}} \mathfrak{2 ^ { 2 }}{ }^{i-1}, \mathfrak{A}^{2^{0}}=\mathfrak{A}$. The radical of a Jordan algebra is the maximal solvable ideal. A Jordan algebra with 0 radical is said to be semi-simple. It has been shown by Albert that any semisimple Jordan algebra of characteristic 0 has an identity and is a direct sum of simple algebras [1, p. 557]. It has been shown recently by Penico that every Jordan algebra of characteristic 0 can be decomposed as $\mathfrak{A}=\subseteq \oplus \mathfrak{N}$ where $\subseteq$ is a semi-simple subalgebra and $\mathfrak{R}$ is the radical [13]. This is the analogue of Wedderburn's "principal theorem" for associative algebras and of the Levi decomposition theorem for Lie algebras. The center \& of a Jordan algebra is the totality of elements $c$ that associate with every pair $a, b$ in $\mathfrak{A}$ in the sense that $[c a b]=[b c a]=[a b c]=0$. This definition can also be used for arbitrary Lie triple systems. It is known that if $\mathbb{E}$ is the center of a simple algebra with an identity then the set $\Re(\mathfrak{E})$ of element $R_{c}, c$ in $\mathfrak{E}$, is the complete set of linear transformations that commute with the multiplications $R_{a}$ (see for example $[9$, p. 239]). It is easy to see that this result holds also for semisimple algebras of characteristic 0 .

We shall now take up the study of the structure of the enveloping Lie and associative algebras of representations of Jordan algebras of characteristic 0 . The results which we shall give can be formulated in terms of the universal algebras. However, we prefer to state them in terms of arbitrary representations.

If $S$ is a representation of $\mathfrak{A}$ we denote the set of representing linear transformations by $S(\mathfrak{H})$ and the enveloping associative algebra by $S(\mathfrak{A})^{*}$. $S(\mathfrak{H})$ is a Lie triple system, a homomorphic image of the associator system of $\mathfrak{A}$. Hence the enveloping Lie algebra $\mathfrak{R}_{S}=S(\mathfrak{H})+[S(\mathfrak{H}), S(\mathfrak{A})]$. We now prove the following theorem.

THEOREM 8.1. Let $\mathfrak{A}$ be a Jordan algebra of characteristic $0, \mathfrak{N}$ its radical,

(5) Levi's theorem states that any finite-dimensional Lie algebra can be expressed as a sum $\Re+\subseteq$ where $\Re$ is the radical (maximal solvable ideal) and $\subseteq$ is semi-simple. A simple proof of this theorem is given in $[6$, p. 686].

(6) The relation $\mathfrak{l}^{\prime}=\mathfrak{l}$ for semi-simple Lie algebras is a consequence of the fact that $\mathfrak{l}$ is a direct sum of simple algebras. 
and $S$ a representation of $\mathfrak{A}$. Then $S(\mathfrak{R})$ is contained in the radical of the enveloping associative algebra $S(\mathfrak{A})$ * of $S(\mathfrak{A})$.

Proof. It has been shown by Albert that $S(\mathfrak{R})^{*}$ is nilpotent [1, p. 551]. Also $S(\mathfrak{N})$ is an ideal in the Lie triple system $S(\mathfrak{U})$. Hence by Theorem 7.1, $\mathfrak{P}=S(\mathfrak{N})+[S(\mathfrak{N}), S(\mathfrak{N})]$ is subinvariant in $\mathfrak{R}_{S}=S(\mathfrak{U})+[S(\mathfrak{U}), S(\mathfrak{U})]$. Since $\mathfrak{B}^{*}=S(\mathfrak{N})^{*}$, Theorem 7.2 shows that $\mathfrak{P}$ is in the radical of $S(\mathfrak{A})^{*}$. Hence $S(\mathfrak{N})$ is in the radical of $S(\mathfrak{H})^{*}$.

COROLlaRY 8.1. If $S$ is a completely reducible representation of a Jordan algebra, then the radical $\mathfrak{N}$ of $\mathfrak{A}$ is contained in the kernel.

Proof. Our assumption implies that $S(\mathfrak{A}) *$ is semi-simple. Hence $S(\mathfrak{N})=0$.

COROLlaRy 8.2. If the notation is as in the theorem, then $S(\mathfrak{N})+[S(\mathfrak{U}), S(\mathfrak{N})]$ is a nilpotent Lie ideal in the enveloping Lie algebra $\mathfrak{R}_{S}$ of $\mathfrak{S}$.

Proof. $S(\mathfrak{N})+[S(\mathfrak{A}), S(\mathfrak{N})]$ is an ideal in $\mathfrak{R}_{S}$ by Theorem 7.1. Moreover, the enveloping associative algebra of $S(\mathfrak{N})+[S(\mathfrak{A}), S(\mathfrak{N})]$ is in the radical of $S(\mathfrak{U}) *$. Hence this ideal is a nilpotent Lie ideal.

If $\mathfrak{T}$ is a Lie triple system contained in a Lie algebra, then it is easy to see that $[\mathfrak{T} \mathfrak{T}]$ is a subalgebra of the Lie algebra. In particular $[S(\mathfrak{H}), S(\mathfrak{H})]$ is a subalgebra of $\mathfrak{R}_{S}$. It is easy to see also that $[S(\mathfrak{U}), S(\mathfrak{N})]$ is an ideal in $[S(\mathfrak{U}), S(\mathfrak{A})]$. The enveloping associative algebra of this ideal is nilpotent. Hence we have the following corollary.

Corollary 8.3. $[S(\mathfrak{A}), S(\mathfrak{N})]$ is a nilpotent Lie ideal in the Lie algebra $[S(\mathfrak{A}), S(\mathfrak{A})]$.

If $R$ denotes the regular representation, then it is known that the elements of $[R(\mathfrak{A}), R(\mathfrak{A})]$ are derivations. These derivations have been called inner and the subalgebra $[R(\mathfrak{H}), R(\mathfrak{A})]$ is an ideal in the derivation algebra $\mathfrak{D}$ of $\mathfrak{A}[11$, p. 867$]$. Corollary 8.3 shows that $[R(\mathfrak{U}), R(\mathfrak{N})]$ is a nilpotent ideal in the algebra of inner derivations. The theorem itself gives the following result which is needed later.

CoROllaRY 8.4. Every transformation belonging to $[R(\mathfrak{A}), R(\mathfrak{N})]$ is a nilpotent derivation in $\mathfrak{A}$.

We consider next the enveloping algebras of representations of semisimple Jordan algebras. Since any such algebra is a direct sum of simple algebras, the set $R(\mathfrak{U})$ and its enveloping Lie algebra $\mathfrak{R}_{R}$ are completely reducible. It follows that $\mathfrak{R}_{R}$ is a direct sum of its center and its derived algebra $\mathfrak{R}_{R}^{\prime}$ and that $\mathbb{R}_{R}^{\prime}$ is semi-simple [8, p. 878]. Also we have noted that the center is $R(\mathcal{E})$. Hence we have the decomposition $\mathfrak{R}_{R}=\mathfrak{R}_{R}^{\prime} \oplus R(\mathfrak{C})$. On the other hand, since $\mathfrak{A}$ has an identity, $R(\mathfrak{U}) \cap[R(\mathfrak{U}), R(\mathfrak{U})]=0$. Hence $\mathfrak{R}_{R}=R(\mathfrak{U}) \oplus[R(\mathfrak{U}), R(\mathfrak{U})]$ and 


$$
\mathfrak{R}_{R}^{\prime}=[[R(\mathfrak{Y}), R(\mathfrak{A})], R(\mathfrak{Y})] \oplus[R(\mathfrak{I}), R(\mathfrak{U})]
$$

It follows that

$$
\mathfrak{R}_{R}=[[R(\mathfrak{U}), R(\mathfrak{H})], R(\mathfrak{U})] \oplus R(\mathfrak{S}) \oplus[R(\mathfrak{U}), R(\mathfrak{U})]
$$

and

$$
R(\mathfrak{U})=[[R(\mathfrak{A}), R(\mathfrak{U})], R(\mathfrak{U})] \oplus R(\mathfrak{S}) .
$$

Since $\mathfrak{A}$ has an identity, the imbedding $R$ is an isomorphism of the associator system $\mathfrak{A}$ onto $R(\mathfrak{U})$. Accordingly (8.3) gives the decomposition

$$
\mathfrak{U}=\mathfrak{A}^{\prime} \oplus \mathfrak{C},
$$

where $\mathfrak{U}^{\prime}$ is the space spanned by the associators $\left({ }^{7}\right)$. In general, if $\mathfrak{T}$ is any Lie triple system, then the space $\mathfrak{T}^{\prime}$ spanned by the products $[a b c]$ is an ideal in $\mathfrak{T}$. We shall call this ideal the derived system of $\mathfrak{T}$. Corresponding to (8.4) we can rewrite (8.3) as

$$
R(\mathfrak{A})=R\left(\mathfrak{U}^{\prime}\right) \oplus R(\mathfrak{C}) .
$$

Also since $\mathfrak{R}_{R}=R(\mathfrak{U}) \oplus[R(\mathfrak{U}), \quad R(\mathfrak{U})], \quad(8.5)$ shows that $[R(\mathfrak{U}), \quad R(\mathfrak{U})]$ $=\left[R\left(\mathfrak{A}^{\prime}\right), R\left(\mathfrak{A}^{\prime}\right)\right]$. Hence

$$
\mathfrak{R}_{R}^{\prime}=R\left(\mathfrak{A}^{\prime}\right) \oplus\left[R\left(\mathfrak{U}^{\prime}\right), R\left(\mathfrak{A}^{\prime}\right)\right] .
$$

If we recall that $\mathfrak{R}_{R}^{\prime}$ is semi-simple, we can now obtain the following lemma by applying Theorem 7.3.

LEMMA 8.1. If $\mathfrak{A}$ is a semi-simple Jordan algebra of characteristic 0 , then the regular imbedding $R$ is a universal imbedding of the derived system $\mathfrak{A}^{\prime}$ of the associator Lie triple system $\mathfrak{A}$.

We consider next the case of $\mathfrak{A}=\mathfrak{C}$. Then we prove the following lemma.

LEMMA 8.2. The enveloping associative algebra of any representation of a semi-simple associative Jordan algebra of characteristic 0 is semi-simple.

Proof. If $\mathrm{P}$ is an extension of the base field of $\mathfrak{A}$, any representation $S$ of $\mathfrak{A}$ can be extended to one of $\mathfrak{A}_{\mathrm{P}}$. The enveloping associative algebra $S\left(\mathfrak{A}_{\mathrm{P}}\right)^{\text {* }}$ $=S(\mathfrak{A})_{\mathrm{P}}^{*}$. Hence it suffices to prove the lemma for algebraically closed base fields. In this case $\mathfrak{A}$ has a basis of $n$ orthogonal idempotent elements $e_{i}$ and $\sum e_{i}=1$. Also, in view of Theorem 4.1, it suffices to prove the lemma for special representations and for representations such that $S_{1}=1$. Now the result is known for special representations [7, p. 168]. Hence it remains to consider the representations such that $S_{1}=1$. We now note that the defining condition (2.1) implies that $\left[S_{e_{i}} S_{e_{j}}\right]=0$ for $i \neq j$. Equation (2.2) gives $2 S_{e_{i}}^{2} S_{e_{j}}$

(7) This result is due to Schafer [14]. 
$=S_{e_{i}} S_{e_{j}}$ and $S_{e_{i}} S_{e_{j}} S_{e_{k}}=0$ if $i, j, k$ are distinct. It follows that any element of the enveloping associative algebra of $S$ is a linear combination of the eleelements $S_{e_{i}}, S_{e_{i}} S_{e_{j}}, i<j$. Also we have

$$
1=2 S_{1}^{2}-S_{1}=\sum\left(2 S_{e_{i}}^{2}-S_{e_{i}}\right)+4 \sum_{i<j} S_{e_{i}} S_{e_{j}}
$$

Hence

$$
\begin{aligned}
S_{e_{k}}-1=\left(S_{e_{k}}-1\right) 1= & \left(S_{e_{k}}-1\right)\left(2 S_{e_{k}}^{2}-S_{e_{k}}\right) \\
& +\sum_{i \neq k}\left(S_{e_{k}}-1\right)\left(2 S_{e_{i}}^{2}-S_{e_{i}}\right)+4 \sum_{i<j}\left(2 S_{e_{k}}-1\right) S_{e_{i}} S_{e_{j}} \\
= & -\sum_{i \neq k}\left(2 S_{e_{i}}^{2}-S_{e_{i}}\right)+4 \sum_{i<j ; i, j \neq k} S_{e_{i}} S_{e_{j} .}
\end{aligned}
$$

This shows that any element of $S(\mathfrak{A}) *$ is a linear combination of the elements $\left(2 S_{e_{i}}^{2}-S_{e_{i}}\right), 4 S_{e_{i}} S_{e_{j}}, i<j$. Also we can verify that the product of any two distinct elements in this set is 0 . Since their sum is 1 , these elements are or thogonal idempotents. Thus $S(\mathfrak{U})^{*}$ has a basis of orthogonal idempotent elements. Hence $S(\mathfrak{A}) *$ is semi-simple.

We can now prove the following lemma.

Lемма 8.3. If $\mathfrak{A}$ is a semi-simple Jordan algebra of characteristic 0 and $S$ is any representation of $\mathfrak{A}$, then the mapping $R_{a} \rightarrow S_{a}$ can be extended to a homomorphism of the enveloping Lie algebra $\mathfrak{R}_{R}$ onto the enveloping Lie algebra $\mathfrak{R}_{S}$. (Thus, the imbedding $R$ of the Lie triple system $\mathfrak{A}$ is a cover of every imbedding obtained from a representation of the Jordan algebra.)

Proof. This result will follow easily from Lemma 8.1 if it can be proved that $\left[S_{c} S_{a}\right]=0$ for every $c \in \mathbb{C}$ and every $a \in \mathfrak{A}$. Now by Lemma 8.2, $S_{c}$ has simple elementary divisors. Hence the mapping $X \rightarrow\left[X S_{c}\right]$ of the complete algebra of linear transformations has simple elementary divisors and the induced mapping in $\Omega_{S}$ has this property. On the other hand $\left[\left[S_{a} S_{c}\right] S_{c}\right]=S_{[a c c]}=0$ and this implies that $X \rightarrow\left[X S_{c}\right]$ is nilpotent in $\mathfrak{R}_{S}$. It follows that this mapping is 0 . Thus $\left[S_{a} S_{c}\right]=0$ and the lemma is proved.

Since $\mathfrak{R}_{R}^{\prime}$ is semi-simple the theorem on the complete reducibility of the representations of semi-simple Lie algebras implies that the enveloping associative algebra of $\mathfrak{R}_{R}^{\prime}$ and hence of $S\left(\mathfrak{U}^{\prime}\right)$ is semi-simple. Also the algebra $S(\mathfrak{S})^{*}$ is semi-simple and by the proof of the preceding lemma any element of $S(\mathfrak{S})^{*}$ commutes with any element of $S\left(\mathfrak{A}^{\prime}\right)^{*}$. It follows from (8.4) that $S(\mathfrak{U}) *$ is semi-simple. We have therefore proved the following theorem.

THEOREM 8.2. The enveloping associative algebra of any representation of a semi-simple Jordan algebra of characteristic 0 is semi-simple.

Since any semi-simple associative algebra of linear transformations is completely reducible we have the following corollary. 
COROLLARY 8.1. Every representation of a semi-simple Jordan algebra of characteristic 0 is completely reducible.

The two main theorems (8.1 and 8.2) can be combined into a single result by using Penico's theorem that any Jordan algebra can be written as $\mathfrak{U}=\mathfrak{S}+\mathfrak{N}$ where $\mathfrak{S}$ is semi-simple and $\mathfrak{N}$ is the radical. Thus we have the following theorem.

THEOREM 8.3. If $S$ is a representation of an arbitrary Jordan algebra of characteristic 0 , then the radical of $S(\mathfrak{H})^{*}$ is the ideal in $S(\mathfrak{H})^{*}$ generated by the elements $S_{z}, z$ in $\mathfrak{\Re}$. Moreover, if $\mathfrak{A}=\mathfrak{S}+\mathfrak{N}$ where $\mathfrak{S}$ is semi-simple, then $S(\mathfrak{H})^{*}$ $=S(\mathfrak{S})^{*}+\Re$ where $\Re$ is the radical of $S(\mathfrak{A})^{*}$.

Proof. Let $\Re$ denote the ideal in $S(\mathfrak{A})^{*}$ generated by the elements $S_{z}, z$ in $\mathfrak{N}$. Since $\mathfrak{A}=\mathfrak{S}+\mathfrak{N}$, any element of $S(\mathfrak{H})^{*}$ is congruent modulo $\mathfrak{R}$ to an element of $S(\mathfrak{S})^{*}$. Thus $S(\mathfrak{A})^{*}=\Re+S(\mathfrak{S})^{*}$. On the other hand, $\Re$ is contained in the radical of $S(\mathfrak{A})^{*}$ and $S(\mathfrak{S})^{*}$ is semi-simple. It follows that $S(\mathfrak{U})^{*}=\Re+S(\mathfrak{S})^{*}$ and $\Re$ is the radical.

It is easy to see that this theorem includes Theorems 8.1 and 8.2. Also, we can establish the following connection between Penico's theorem and the Levi decomposition of the enveloping Lie algebra $\Omega_{S}$ of $S$ :

THEOREM 8.4. Let $\mathfrak{A}=\Im \oplus \mathfrak{N}$ be a decomposition of the Jordan algebra $\mathfrak{A}$ (of characteristic 0 ) as a direct sum of a semi-simple algebra $\mathfrak{\subseteq}$ and its radical $\mathfrak{N}$ and let $\mathfrak{S}(\mathfrak{S})$ denote the center of $\mathfrak{S}$. Then if $S$ is a representation of $\mathfrak{A}$, the radical of the enveloping Lie algebra $\mathfrak{\Omega}_{S}$ is $S(\mathfrak{S}(\mathfrak{S}))+S(\mathfrak{N})+[S(\mathfrak{N}), S(\mathfrak{N})]$ and $S\left(\mathfrak{\Im}^{\prime}\right)+\left[S\left(\mathfrak{\Im}^{\prime}\right), S\left(\mathfrak{\Im}^{\prime}\right)\right]$ is a semi-simple Levi component of $\mathfrak{R}_{S}$.

The proof of this result can be obtained easily from the foregoing results. We shall therefore omit it.

9. The analogue of Whitehead's first lemma and its applications. As is well known, Whitehead's first lemma on Lie algebras was formulated to give a simple proof of the theorem of complete reducibility of the representations for semi-simple Lie algebras. In this section we shall prove an analogue of Whitehead's result for Jordan algebras. However, we shall reverse the procedure which is customary for Lie algebras and obtain the present result from the theorems of the preceding section. The theorem we wish to prove is the following.

THEOREM 9.1. Let $\mathfrak{M}$ be a Jordan module for a semi-simple Jordan algebra of characteristic 0 and let $a \rightarrow f(a)$ be a linear mapping of $\mathfrak{A}$ into $\mathfrak{M}$ such that

$$
f(a b)=a f(b)+f(a) b .
$$

Then there exist elements $w_{i}$ in $\mathfrak{M}$ and $b_{i}$ in $\mathfrak{A}$ such that

$$
f(a)=\sum\left(w_{i} a\right) b_{i}-w_{i}\left(a b_{i}\right) .
$$


We prove first the following lemma.

Lemma 9.1. If $\mathfrak{A}$ is a semi-simple Jordan algebra of characteristic 0 which contains no associative ideals not 0 , then the subalgebra of $\mathfrak{A}$ generated by the derived system $\mathfrak{A}^{\prime}$ is $\mathfrak{A}$ itself.

Proof. It suffices to prove this for algebraically closed fields and for the case $\mathfrak{A}$ simple. Here the center is one-dimensional, so that by (8.4) dim $\mathfrak{A}^{\prime}$ $=\operatorname{dim} \mathfrak{A}-1$. If the lemma is false, $\mathfrak{A}^{\prime}$ is a subalgebra of $\mathfrak{A}$. Since the center consists of the multiples of 1 this implies that $\mathfrak{A}^{\prime}$ is an ideal, contrary to the simplicity of $\mathfrak{A}$.

Proof of Theorem 9.1. We form the semi-direct sum $\mathfrak{B}=\mathfrak{A}+\mathfrak{M}$ and let $D$ be the linear transformation in $\mathfrak{B}$ such that $\mathfrak{M D}=0$ and $a D=f(a)$ for $a$ in $\mathfrak{A}$. Then by (9.1), $D$ is a derivation in $\mathfrak{B}$ which maps $\mathfrak{A}$ into $\mathfrak{M}$, $\mathfrak{M}$ into 0 . If $a \in \mathfrak{B}$, and $R_{u}$ denotes the multiplication in $\mathfrak{B}$ by $u$, then $\left[R_{u}, D\right]=R_{u D}$. It follows that the mapping $X \rightarrow[X D]$ is a derivation $\tilde{D}$ in the enveloping Lie algebra $\Omega_{R}$ of the $R_{u}$. If $\mathbb{E}$ is the center of $\mathfrak{A}$, then by Theorem 8.4 the radical of $\Omega_{R}$ is

$$
\mathfrak{B}=R(\mathfrak{C})+R(\mathfrak{M})+[R(\mathfrak{A}), R(\mathfrak{M})]
$$

and

$$
\mathfrak{F}=R\left(\mathfrak{H}^{\prime}\right)+\left[R\left(\mathfrak{H}^{\prime}\right), R\left(\mathfrak{H}^{\prime}\right)\right]
$$

is a semi-simple Levi component of $R_{R}$. It is easy to verify that $\mathfrak{R}_{1} \equiv \mathfrak{F}+\mathfrak{Z}_{1}$, where $\mathfrak{Z}_{1}=R(\mathfrak{M})+[R(\mathfrak{R}), R(\mathfrak{M})]$, is a subalgebra of $\mathfrak{R}_{R}$ and that $\left[\mathfrak{Z}_{1} \mathfrak{Z}_{1}\right]=0$. Evidently $\mathfrak{Z}_{1}$ is the radical of $\mathfrak{R}_{1}$. Since $\mathfrak{R}_{R}=R(\mathfrak{H})+R(\mathfrak{M})+[R(\mathfrak{H}), R(\mathfrak{M})], \tilde{D}$ maps $\mathfrak{R}_{R}$ into $R(\mathfrak{M})$. Hence $\tilde{D}$ induces a derivation in $\mathfrak{R}_{1}$ that maps the semisimple algebra $\mathfrak{F}$ into $Z_{1}, Z_{1}$ into 0 . Since $\left[Z_{1} \not_{1}\right]=0$ we can use Whitehead's first lemma for Lie algebras $\left[16 ; 6\right.$, p. 682] to conclude that there is a $U \in Z_{1}$ such that $[X D]=[X U]$ holds for all $X \in \mathfrak{R}_{1}$. Now $U=R_{z}+\sum\left[R_{w_{i}} R_{b_{i}}\right], z$, $w_{i} \in \mathfrak{M}, b_{i} \in \mathfrak{A}$. Hence for any $a^{\prime} \in \mathfrak{Y}^{\prime}$,

$$
R_{a^{\prime} D}=\left[R_{a^{\prime}} D\right]=\left[R_{a^{\prime}} U\right]=\left[R_{a^{\prime}} R_{z}\right]+\sum\left[R_{a^{\prime}}\left[R_{w_{i}} R_{b_{i}}\right]\right] .
$$

Hence

$$
a^{\prime} D=1 R_{a^{\prime} D}=a^{\prime} \sum\left[R_{w_{i}} R_{b_{i}}\right] .
$$

This shows that $D$ coincides with the inner derivation $I=\sum\left[R_{w_{i}} R_{b_{i}}\right]$ on the derived system $\mathfrak{I}^{\prime}$. It follows that $D=I$ on the subalgebra generated by $\mathfrak{X}^{\prime}$. Hence, if $\mathfrak{A}$ contains no associative ideals not $0, D=I$.

Now suppose that $\mathfrak{A}$ has associative ideals. Then we wish to show that $D$ can be expressed in the form $\sum\left[R_{w_{i}} R_{b_{i}}\right], w_{i}$ in $\mathfrak{M}, b_{i}$ in $\mathfrak{A}$. It suffices to prove this under the assumption that the base field is algebraically closed. In this case the associative simple components in the direct decomposition of $\mathfrak{A}$ into simple ideals are one-dimensional. We shall prove our assertion by induction 
on the number of these associative simple components. The preceding argument shows that the result holds if there are no such components. Hence assume that it holds for $r$. Then we can find an inner derivation $I_{r}=\sum\left[R_{w_{i}} R_{b_{i}}\right]$, $w_{i}$ in $\mathfrak{M}, b_{i}$ in $\mathfrak{A}$, such that $D_{r} \equiv D-I_{r}$ maps all the nonassociative simple components into 0 and also the first $r$ associative simple components into 0 . Let $\left(e_{r+1}\right)$ be the $(r+1)$ st associative simple component. We may suppose that $e_{r+1}^{2}=e_{r+1}$. Hence $e_{r+1}\left(e_{r+1} D_{r}\right)=e_{r+1} D_{r} / 2$. Then

$$
e_{r+1} D_{r}=e_{r+1}\left(4\left[R_{e_{r+1}} R_{e_{r+1} D_{r}}\right]\right) \text {. }
$$

Moreover, if $u$ is in the sum of the preceding components, then

$$
u\left[R_{e_{r+1}} R_{e_{r+1} D_{r}}\right]=\left(u e_{r+1}\right)\left(e_{r+1} D_{r}\right)-\left(u\left(e_{r+1} D_{r}\right)\right) e_{r+1}=-\left(u\left(e_{r+1} D_{r}\right)\right) e_{r+1} .
$$

Since $u e_{r+1}=0, \quad\left(u D_{r}\right) e_{r+1}+u\left(e_{r+1} D_{r}\right)=0$ and since $u D_{r}=0, u\left(e_{r+1} D_{r}\right)=0$. Hence $u\left[R_{e_{r+1}} R_{e_{r+1} D_{r}}\right]=0$. Thus $D_{r+1}=D_{r}-4\left[R_{e_{r+1}} R_{e_{r+1} D_{r}}\right]$ maps the nonassociative simple components into 0 , and the first $r+1$ associative simple components into 0 . This proves that $D=\sum\left[R_{w_{i}} R_{b_{i}}\right], w_{i}$ in $\mathfrak{M}$ and $b_{i}$ in $\mathfrak{A}$. Hence

$$
f(a)=a D=\sum\left(\left(w_{i} a\right) b_{i}-w_{i}\left(a b_{i}\right)\right)
$$

as required.

As in the case of Lie and associative algebras, it is easy to see that Theorem 9.1 is equivalent to the following result:

THEOREM 9.2. If $\mathfrak{A}$ is a semi-simple subalgebra of any Jordan algebra $\mathfrak{B}$ of characteristic 0 , then any derivation of $\mathfrak{A}$ into $\mathfrak{B}$ can be extended to an inner derivation of $\mathfrak{B}$ (cf. [6, p. 689]).

By a derivation of $\mathfrak{A}$ into $\mathfrak{B}$ we mean a linear mapping $a \rightarrow a D$ of $\mathfrak{A}$ into $\mathfrak{B}$ such that $\left(a_{1} a_{2}\right) D=\left(a_{1} D\right) a_{2}+a_{1}\left(a_{2} D\right)$ holds for every $a_{1}, a_{2}$ in $\mathfrak{A}$. The proof of this theorem is identical with that of the corresponding Lie result and will therefore be omitted.

We shall now apply Theorem 9.1 to the study of the semi-simple subalgebras of a Jordan algebra. We recall first that if $\mathfrak{A}$ is an arbitrary nonassociative algebra of characteristic 0 and $D$ is a nilpotent derivation in $\mathfrak{A}$, then

$$
G=\exp D=1+D+\frac{D^{2}}{2 !}+\frac{D^{3}}{3 !}+\cdots
$$

is an automorphism of $\mathfrak{A}$. We shall say that two subalgebras of $\mathfrak{A}$ are strictly conjugate if there exists an automorphism of the form $G_{1} G_{2} \cdots G_{k}, G_{i}$ $=\exp D_{i}, D_{i}$ a nilpotent derivation, mapping one of the algebras onto the other. The main result that we shall prove can now be stated as follows:

THEOREM 9.3. Let $\mathfrak{A}$ be an arbitrary Jordan algebra of characteristic 0 and let $\mathfrak{A}=\mathfrak{S}+\mathfrak{N}$ where $\mathfrak{S}$ is semi-simple and $\mathfrak{N}$ is the radical. Then any semi-simple 
subalgebra $\mathfrak{F}$ of $\mathfrak{A}$ is strictly conjugate to a subalgebra of $\mathfrak{S}$.

Proof. If $f \in \mathfrak{F}$ we write $f=\sigma(f)+\nu(f)$ where $\sigma(f) \in \mathfrak{S}$ and $\nu(f) \in \mathfrak{N}$. Then the mappings $f \rightarrow \sigma(f), f \rightarrow \nu(f)$ are linear. Moreover, if $f_{1}, f_{2} \in \mathfrak{F}$ then

$$
\begin{aligned}
f_{1} f_{2} & =\sigma\left(f_{1} f_{2}\right)+\nu\left(f_{1} f_{2}\right) \\
& =\sigma\left(f_{1}\right) \sigma\left(f_{2}\right)+\nu\left(f_{1}\right) \sigma\left(f_{2}\right)+\sigma\left(f_{1}\right) \nu\left(f_{2}\right)+\nu\left(f_{1}\right) \nu\left(f_{2}\right) .
\end{aligned}
$$

Hence

$$
\begin{aligned}
& \sigma\left(f_{1} f_{2}\right)=\sigma\left(f_{1}\right) \sigma\left(f_{2}\right), \\
& \nu\left(f_{1} f_{2}\right)=\nu\left(f_{1}\right) \sigma\left(f_{2}\right)+\sigma\left(f_{1}\right) \nu\left(f_{2}\right)+\nu\left(f_{1}\right) \nu\left(f_{2}\right) .
\end{aligned}
$$

The first of these equations shows that $\sigma$ is a homomorphism of $\mathfrak{F}$ into $\mathfrak{S}$. Hence, if $S_{f} \equiv R_{\sigma(f)}$, then $f \rightarrow S_{f}$ is a representation of $\mathfrak{F}$ and this representation induces a representation in $\mathfrak{N}$.

We now make use of a certain chain of ideals that has been defined by Penico [13]. We set $\mathfrak{N}_{1}=\mathfrak{N}$ and define $\mathfrak{N}_{k}$ inductively as $\mathfrak{N}_{k-1}^{2}+\mathfrak{A} \mathfrak{N}_{k-1}^{2}$. Then $\mathfrak{N}_{k}$ is an ideal in $\mathfrak{A}$ and $\mathfrak{N}_{1} \supset \mathfrak{N}_{2} \supset \mathfrak{N}_{3} \supset \ldots$. Now assume that the $\nu(f) \in \mathfrak{N}_{k}$. This is equivalent to saying that $\mathfrak{F} \subseteq \subseteq+\mathfrak{N}_{k}$. Then we shall show that there exists an automorphism $G$ of the form $\exp D$, where $D$ is in the radical of the enveloping associative algebra of $R(\mathfrak{A})$, such that $\mathfrak{F}^{G} \subseteq \mathfrak{S}+\mathfrak{N}_{k+1}$. Since, in any case, $\mathfrak{F} \subset \mathfrak{S}+\mathfrak{N}_{1}$, this will prove the theorem by induction on $k$.

Since the $\mathfrak{N}_{i}$ are ideals, $S$ induces representations in these spaces. Let $\bar{S}$ denote the induced representation in $\mathfrak{N}_{k} / \mathfrak{N}_{k+1}$ and let $\bar{\nu}(f)$ denote the coset of $\nu(f)$ modulo $\mathfrak{N}_{k+1}$. Then by (9.7)

$$
\bar{\nu}\left(f_{1} f_{2}\right)=\bar{\nu}\left(f_{1}\right) f_{2}+f_{1} \bar{\nu}\left(f_{2}\right)
$$

holds in the module $\mathfrak{N}_{k} / \mathfrak{N}_{k+1}$. Hence by Theorem 9.1 there exist elements $\bar{w}_{i}$ in $\mathfrak{N}_{k} / \mathfrak{N}_{k+1}$ and $b_{i}$ in $\mathfrak{F}$ such

$$
\bar{\nu}(f)=\sum\left(\left(\bar{w}_{i} f\right) b_{i}-\bar{w}_{i}\left(f b_{i}\right)\right) .
$$

It follows that

$$
\nu(f) \equiv \sigma(f) \sum\left[R_{w_{i}} R_{\sigma\left(b_{i}\right)}\right]\left(\bmod \mathfrak{N}_{k+1}\right) .
$$

The mapping $D=\sum\left[R_{w_{i}} R_{\sigma\left(b_{i}\right)}\right]$ is a derivation belonging to the radical of $R(\mathfrak{A})^{*}$. Hence $G=\exp (-D)$ is an automorphism. Now

$$
f^{G}=f-f D+\frac{1}{2 !} f D^{2}-\cdots=\sigma(f)+\nu(f)-\sigma(f) D-\nu(f) D+\cdots .
$$

The terms omitted contain $D^{2}, D^{3}, \cdots$. Since the elements $w_{i} \in \mathfrak{N}_{k}$, the form of $D$ shows that these terms belong to $\mathfrak{N}_{k+1}$. Also $\nu(f) D \in \mathfrak{N}_{k+1}$ and, by (9.8), $\nu(f)-\sigma(f) D \in \mathfrak{N}_{k+1}$. Hence $\mathfrak{F}^{G} \subseteq \subseteq+\mathfrak{N}_{k+1}$ as required. This completes the proof.

We remark that the automorphism which we have used is of the form 
$\exp D_{1} \exp D_{2} \cdots \exp D_{r}$ where the $D_{i}$ are in the radical of the enveloping associative algebra of $R(\mathfrak{A})$. Now if $D_{1}$ and $D_{2}$ are derivations whose enveloping associative algebra is nilpotent, then the Campbell-Hausdorff formula

$$
\exp D_{1} \exp D_{2}=\exp \left(D_{1}+D_{2}+\frac{1}{2}\left[D_{1} D_{2}\right]+\cdots\right)
$$

is valid (see for example $[2$, p. 81$]$ ). This shows that the conjugacy of $\mathfrak{F}$ to a subalgebra of $\subseteq$ can be given by an automorphism of the form $\exp D, D$ in the radical of $R(\mathfrak{H})^{*}$.

An immediate consequence of the theorem is the following corollary.

Corollary 9.1. Any semi-simple subalgebra of $\mathfrak{A}$ can be embedded in a semisimple subalgebra $\mathfrak{S}_{1}$ such that $\mathfrak{A}=\mathfrak{S}_{1}+\mathfrak{R}$.

\section{BIBLIOGRAPHY}

1. A. A. Albert, $A$ structure theory for Jordan algebras, Ann. of Math. vol. 48 (1947) pp. 446-467.

2. G. Birkhoff, Analytical groups, Trans. Amer. Math. Soc. vol. 43 (1938) pp. 61-101.

3. G. Birkhoff and P. Whitman, Representations of Jordan and Lie algebras, Trans. Amer. Math. Soc. vol. 61 (1949) pp. 116-136.

4. S. Eilenberg, Extensions of general algebras, Annales de la Société Polonaise de Mathématique vol. 21 (1948) pp. 125-134.

5. Harish-Chandra, On the radical of a Lie algebra, Proceedings of the American Mathematical Society vol. 1 (1950) pp. 14-17.

6. G. Hochschild, Semi-simple algebras and generalized derivations, Amer. J. Math. vol. 64 (1941) pp. 677-694.

7. F. D. Jacobson and N. Jacobson, Classification and representation of semi-simple Jordan algebras, Trans. Amer. Math. Soc. vol. 65 (1949) pp. 141-169.

8. N. Jacobson, Rational methods in the theory of Lie algebras, Ann. of Math. vol. 36 (1935) pp. $875-881$.

9. - Structure theory of simple rings without finiteness assumptions, Trans. Amer. Math. Soc. vol. 57 (1945) pp. 228-245.

10. —-, Lie and Jordan triple systems, Amer. J. Math. vol. 71 (1949) pp. 149-170.

11. - Derivation algebras and multiplication algebras of semi-simple Jordan algebras, Ann. of Math. vol. 50 (1949) pp. 866-874.

12. A. Malcev, On the representation of an algebra as a direct sum of the radical and a semisimple algebra, C. R. (Doklady) Acad. Sci. URSS vol. 36 (1942) pp. 42-45.

13. A. J. Penico, The Wedderburn principal theorem for Jordan algebras, Trans. Amer. Math. Soc. vol. 70 (1951) pp. 404-421.

14. R. D. Schafer, $A$ theorem on the derivations of Jordan algebras, Proceedings of the American Mathematical Society vol. 2 (1951) pp. 290-294.

15. E. V. Schenkman, $A$ theory of subinvariant Lie algebras, to appear in Amer. J. Math.

16. J. H. C. Whitehead, Certain equations in the algebra of a semi-simple infinitesimal group, Quart. J. Math. Oxford Ser. vol. 8 (1937) pp. 220-237.

17. E. Witt, Treue Darstellung Liescher Ringe, Journal für Mathematik vol. 177 (1937) pp. 152-160.

YALE UNIVERSITY,

New Haven, Conn. 